The Astrophysical JouRnal, 495:458-467, 1998 March 1

(C) 1998. The American Astronomical Society. All rights reserved. Printed in U.S.A.

\title{
ASTEROSEISMOLOGY OF A STAR COOLED BY NEUTRINO EMISSION: THE PULSATING PRE-WHITE DWARF PG $0122+200$
}

\author{
M. S. O’Brien, ${ }^{1}$ G. Vauclatr, ${ }^{2}$ S. D. Kawaler, ${ }^{1}$ T. K. Watson, ${ }^{3}$ D. E. Winget, ${ }^{4}$ R. E. Nather, ${ }^{4}$ M. Montgomery, ${ }^{4}$ \\ A. Nitta, ${ }^{4}$ S. J. Kleinman, ${ }^{5}$ D. J. Sullivan, ${ }^{6}$ X. J. Jiang, ${ }^{7}$ T. M. K. Marar, ${ }^{8}$ S. Seetha, ${ }^{8}$ B. N. Ashoka, ${ }^{8}$ \\ J. Bhattacharya, ${ }^{8}$ E. M. Leibowitz, ${ }^{9}$ S. Hemar, ${ }^{9}$ P. Ibbetson, ${ }^{9}$ B. Warner, ${ }^{10}$ L. VAn ZyL, ${ }^{10}$ \\ P. MoskaliK, ${ }^{11}$ S. Zoła, ${ }^{12,13}$ G. PAJdosz, ${ }^{12}$ J. KrzesińsKi, ${ }^{12}$ N. Dolez, ${ }^{2}$ M. CheVreton, ${ }^{14}$ \\ J.-E. Solheim, ${ }^{15,16}$ T. Thomassen, ${ }^{15}$ S. O. KePler, ${ }^{17,18}$ O. Giovannini, ${ }^{17}$ \\ J. L. Provencal, ${ }^{19}$ M. A. Wood ${ }^{20}$ AND J. C. Clemens ${ }^{21}$ \\ Received 1997 August 14; accepted 1997 October 9
}

\begin{abstract}
Observation of $g$-mode pulsations in the variable pre-white dwarf (GW Virginis) stars provides a unique means to probe their interiors and to study the late stages of stellar evolution. Multisite campaigns have in several cases proved highly successful in decoding pre-white dwarf light curves. Three previous attempts to untangle the pulsation spectrum of the coolest GW Virginis star, PG $0122+200$, confirmed the existence of multiple $g$-modes but left the fundamental period spacing and therefore the star's mass and luminosity in doubt. We present an analysis based on new observations of PG $0122+200$ obtained during a Whole Earth Telescope (WET) campaign conducted in the fall of 1996. Although our coverage was, because of bad weather, far poorer than in previous WET campaigns, we confirm the previous result that PG $0122+200$ rotates once in $1.6 \pm 0.1$ days. The most likely period spacing supported by the data implies a mass of $0.69 \pm 0.03 M_{\odot}$. Based on the best seismology we can currently do, the cooling of PG $0122+200$ is dominated by neutrino losses. This is not true for all prewhite dwarf stars and makes PG $0122+200$ the prime candidate for learning useful physics. Constraints placed on the cooling rate of PG $0122+200$ by future measurement of $d \Pi / d t$ could provide a unique test of the standard theory of lepton interactions in the (experimentally unexplored) region of phase-space appropriate to pre-white dwarf interiors.
\end{abstract}

Subject headings: elementary particles — stars: individual (PG 0122+200) — stars: interiors stars: pulsars: general — stars: white dwarfs

\section{INTRODUCTION}

The variable star PG $0122+200$ (BB Psc, hereafter PG $\left.0122 ; m_{b}=16.13\right)$ is a hot, luminous star of the PG 1159 spectroscopic class. The PG 1159 stars, about a third of which are intrinsic variables like PG 0122, represent a transitory phase of stellar evolution between the asymptotic giant branch and the white dwarf cooling track. Most of them show evidence of recent mass loss in the form of surrounding nebulosity; this includes one of the largest - and perhaps oldest-planetary nebulae ever found, RXJ 2117+ 34 (Appleton, Kawaler, \& Eitter 1993). From an astrophysical point of view, pre-white dwarf structure reveals important information about the deaths of ordinary stars and about the formation of white dwarf stars. The physics of their interiors is also interesting on its own account. Specifically, the fundamental physics controlling their structure is that of weak-force (leptonic) interactions: neutrino emission quickly overtakes photon luminosity as the primary source of energy loss in the coolest members of the class (Iben \& Tutukov 1984; also see Kawaler, Hansen, \& Winget 1985).

The brightness fluctuations seen in variable PG 1159 stars are an effect of nonradial $g$-mode pulsation at resonant eigenfrequencies within the stellar interior. The pulsation probes that interior. Consecutive high radial overtone $(n \gg 1)$ eigenmodes of a given spherical harmonic order $\ell$

\footnotetext{
${ }^{1}$ Department of Physics and Astronomy, Iowa State University, Ames, IA 50011; msobrien@iastate.edu.

${ }_{2}^{2}$ Observatoire Midi-Pyrenees, 14 Avenue E. Belin, 31400 Toulouse, France.

${ }^{3}$ International Institute of Theoretical and Applied Physics, 123 Office and Lab, Iowa State University, Ames, IA 50011.

${ }^{4}$ Department of Astronomy, University of Texas, Austin, TX 78712.

${ }^{5}$ California Institute of Technology, Pasadena, CA 91125.

${ }^{6}$ Department of Physics, Victoria University, Box 600, Wellington, New Zealand.

${ }^{7}$ Beijing Observatory, Chinese Academy of Sciences, Beijing 100080, China.

${ }^{8}$ Indian Space Research Organization, Technical Physics Division, ISRO Satellite Centre, Airport Road, Bangalore 560017, India.

${ }^{9}$ Department of Physics and Astronomy, University of Tel Aviv, Ramat Aviv, Tel Aviv 69978, Israel.

${ }^{10}$ Department of Astronomy, University of Cape Town, Rondebosch 7700, Cape Province, South Africa.

11 Copernicus Astronomical Center, ul. Bartycka 18, 00-716 Warsaw, Poland.

${ }^{12}$ Mount Suhora Observatory, Cracow Pedagogical University, ul. Podchorążych 2, 30-084 Kraków, Poland.

${ }^{13}$ Astronomical Observatory, Jagiellonian University, ul. Orla 171, 30-244 Kraków, Poland.

${ }^{14}$ Observatoire de Paris-Meudon, F-92195 Meudon, Principal Cedex, France.

${ }^{15}$ Institutt for Matematiske Realfag, Universitet i Tromso, 9000 Tromso, Norway.

${ }^{16}$ Guest Observer, Isaac Newton Telescope, Roque de los Muchachos, La Palma, Canaries.

${ }^{17}$ Instituto de Fisica, Universidade Federal do Rio Grande do Sul, 91501-970 Porto Alegre-RS, Brazil.

${ }^{18}$ Guest Observer, Observatorio do Pico dos Dias, LNA/CNPq, Brazil.

${ }^{19}$ University of Delaware, Department of Physics and Astronomy, Sharp Laboratory, Newark, DE 19716.

${ }^{20}$ Department of Physics and Space Science, Florida Institute of Technology, 150 West University Boulevard, Melbourne, FL 32901.

${ }^{21}$ Palomar Observatory, 105-24, California Institute of Technology, Pasadena, CA 91125.
} 
are approximately equally spaced in period. Periodic deviations from equal spacing record the effects of transition regions between different layers in the chemically stratified pre-white dwarf stars (Kawaler 1987). The observed pattern of modes allows us to pare down the large set of stellar models that satisfactorily explain observed surface properties (brightness, temperature, gravity, chemical makeup) to a small subset with a very narrow range of mass, luminosity, and internal structure (see, for instance, Kawaler \& Bradley 1994). In addition, slow stellar rotation splits each mode into $2 \ell+1$ components spaced equally in frequency, though a weak magnetic field can make the frequency splitting uneven about the central peak. When observed, this frequency splitting, along with the period distribution, lets us decode the mode structure. However, in practice, the pulsation spectra are complex-a single star can show dozens to over a hundred separate periodicities - and need long, nearly continuous data sets to resolve modes closely spaced in frequency and to reduce aliasing caused by gaps in the data. Aliasing, in particular, can hopelessly complicate interpretation of single-site data sets.

The solution to these problems is to observe with a multisite telescope network such as the Whole Earth Telescope (WET; Nather et al. 1990). So far, three pre-white dwarfs have been studied successfully in this way: PG 1159-035 (Winget et al. 1991) and PG 2131+066 (Kawaler et al. 1995), both observed with the WET, and the central star of the planetary nebula NGC 1501 (Bond et al. 1996), observed with a network of CCD photometers. With the pulsation spectra of three stars in hand, we now begin to address detailed questions about the variable pre-white dwarfs as a class of objects. The prospect of adding a fourth star-PG 0122 - to this list makes the study of its periodicities and structure even more exciting.

Pulsating PG 1159 stars with periods less than 1000 $\mathrm{s}$ - and no nebulae - form the GW Virginis class, of which PG $1159-035$ is the prototype. With an effective temperature of 75,000 K (Dreizler, Werner, \& Heber 1995), PG 0122 is the coolest known GW Virginis star, and it currently defines the red edge of the instability strip. After discovery of its photometric variations by Bond \& Grauer (1987), three separate efforts were made to unravel its complex light curve. Hill, Winget, \& Nather (1987) observed the star on four consecutive nights in late 1986 with the $2.1 \mathrm{~m}$ telescope at McDonald Observatory and tentatively identified eight separate pulsation modes between 300 and $700 \mathrm{~s}$. They further proposed a mean period spacing of $16.4 \mathrm{~s}$, which implied a mass of $\sim 0.7 M_{\odot}$, based on models then available. However, their analysis rested on the technique of averaging together the Fourier transforms of separate nightly data sets, a procedure that, as it turned out, left unresolved the rotational splitting later found to exist in several individual modes of this star. Compelling mode identification was hence impossible for Hill et al.

A decade later, armed with hindsight created by successes with PG 1159 and PG 2131, O'Brien et al. (1996; hereafter OCKD) reanalyzed the data taken by Hill et al. OCKD identified 13 individual frequencies, comprising singlets, doublets, and one clear triplet, in the transform of the full Hill et al. data set. We identified four probable $\ell=1$ modes and one possible $\ell=2$ mode and found strong evidence for an $\ell=1$ frequency splitting of $3.6 \mu \mathrm{Hz}$ with a mean period spacing of $21.2 \mathrm{~s}$. These numbers imply a rotation rate of $\sim 1.6$ days and a mass of $0.66-0.72 M_{\odot}$ for PG 0122 .
However, OCKD made significant use of the technique of prewhitening in order to separate the presumed real peaks from the effects of severe aliasing introduced by daily gaps in the data. Thus their mode identifications were tentative, and other possible "solutions" to this data set could not be ruled out. In particular, though the strongest evidence for the 1.6 day rotation period was found in the transform's dominant multiplet at $400 \mathrm{~s}$, a rotation rate of 0.8 days accounted for the peaks in this triplet mode almost equally well if it was assumed that the third largest mode found in the triplet was really an alias of the "true" peak $11.6 \mu \mathrm{Hz}$ away.

Meanwhile, in 1990 October, a separate group organized a bilongitudinal campaign to observe PG 0122 with the Steward Mount Lemmon $1.5 \mathrm{~m}$ telescope, the Steward Mount Bigelow $1.5 \mathrm{~m}$ telescope, and the Roque de los Muchachos Nordic Optical $2.5 \mathrm{~m}$ Telescope. Based on 74.7 $\mathrm{hr}$ of data obtained over 11 days, Vauclair et al. (1995; hereafter VPG) report evidence for a frequency splitting indicative of a $\sim 1.8$ day rotation rate for PG 0122 , consistent within errors in the frequency determinations with the rotation rate deduced by OCKD. Unfortunately, their attempt to fit a pattern of equal spacing to the periods was frustrated by the apparent absence of some low-amplitude modes separately identified by OCKD. Of the 13 peaks identified by OCKD in the transform of the 1986 data, only seven were identified in the 1990 data taken by VPG. VPG suggest a period spacing of $\sim 16 \mathrm{~s}$, basing this result on two different arguments. The first argument derives from the suggestion of Hill et al. that, given similar structure, PG 0122 and PG 1159 should have period spacings in the same ratio as their respective dominant periods. However, the resulting estimated period spacing for PG 0122 requires a mass of at least $0.7 M_{\odot}$, far greater than the $0.58 M_{\odot}$ of PG 1159 , implying that the two stars have significantly different structure. Their second argument is based on the observed periods of three multiplets that VPG tentatively identify with the order $\ell=1$. They express doubts as to the exact nature of one of the modes, however, suggesting that an $\ell=2$ identification might also explain the observed peaks. OCKD independently identified the problematic multiplet as a likely $\ell=2$ mode.

The three attempts at decryption of the PG 0122 light curve discussed above were of necessity incomplete, inconclusive, and very tantalizing. They strongly suggested the need for a more comprehensive and concerted observational campaign to resolve the issues of mode identification and hence rotational splitting and period spacing. Indisputable measurement of these quantities would, in conjunction with theoretical stellar models, pinpoint the mass and luminosity of PG 0122 and reveal much about its evolutionary status. In addition, the mass of PG 0122 helps determine the role that neutrino emission plays as a source of cooling (O'Brien \& Kawaler 1998). If this mass is indeed large - one point on which all three prior analyses concurthen according to stellar models, neutrino luminosity dominates the energy output of PG 0122 as in no other pre-white dwarf star known (O’Brien \& Kawaler 1998). This neutrino cooling should produce secular changes in the pulsation periods of PG 0122. Such changes should be measurable.

The revealing but somewhat ambiguous results of singleand double-site observation of PG 0122 made it an ideal subject for observation with the WET. It was the primary 
TABLE 1

OBSERVATIONS OF PG 0122 FOR THE WET COVERAGE IN 1996

\begin{tabular}{|c|c|c|c|c|}
\hline Telescope & Run Name & $\begin{array}{c}\text { Date } \\
\text { (1996 Sep) }\end{array}$ & $\begin{array}{l}\text { Start Time } \\
\text { (UT) }\end{array}$ & $\begin{array}{l}\text { Run Length } \\
\text { (h:mm:ss) }\end{array}$ \\
\hline \multirow[t]{2}{*}{ Pic du Midi $2 \mathrm{~m} . . . \ldots \ldots$} & gv-0476 ${ }^{a}$ & 7 & $11: 46: 00$ & $4: 18: 50$ \\
\hline & gv-0478 & 9 & $00: 16: 00$ & $3: 46: 50$ \\
\hline La Palma NOT ............ & jestt06 & 9 & $03: 37: 00$ & $2: 19: 00$ \\
\hline \multirow[t]{2}{*}{ Pic du Midi 2 m.................. } & gv-0479 ${ }^{a}$ & 10 & 00:03:01 & $0: 25: 50$ \\
\hline & gv-0480 & 10 & $03: 32: 30$ & $3: 32: 30$ \\
\hline \multirow[t]{2}{*}{ McDonald $2.1 \mathrm{~m} . . . . . . .}$. & an-0049 & 10 & $05: 58: 30$ & $3: 18: 40$ \\
\hline & an-0050 & 10 & $10: 36: 50$ & $1: 21: 40$ \\
\hline BAO $2.2 \mathrm{~m} \ldots \ldots \ldots$ & bao- $0030^{a}$ & 10 & $18: 39: 30$ & $2: 04: 00$ \\
\hline Pic du Midi $2 \mathrm{~m} . .$. & gv-0482 & 13 & $00: 16: 01$ & $4: 00: 10$ \\
\hline La Palma NOT..... & jestt08 ${ }^{\mathrm{a}}$ & 13 & $00: 23: 00$ & $5: 29: 30$ \\
\hline \multirow[t]{2}{*}{ Pic du Midi $2 \mathrm{~m} . . . . . . . . .}$. & gv-0483 & 14 & $01: 46: 02$ & $2: 20: 20$ \\
\hline & gv- $0485^{a}$ & 15 & $00: 27: 02$ & $3: 41: 20$ \\
\hline McDonald $2.1 \mathrm{~m}$........... & an- $-0051^{\mathrm{a}}$ & 15 & $05: 31: 10$ & $2: 47: 30$ \\
\hline BAO $2.2 \mathrm{~m} \ldots \ldots \ldots \ldots \ldots$ & bao- $0033^{\mathrm{a}}$ & 15 & $17: 04: 30$ & $2: 31: 30$ \\
\hline Pic du Midi 2 m........... & gv-0486 & 15 & $23: 13: 03$ & $5: 07: 00$ \\
\hline \multirow[t]{3}{*}{ McDonald $2.1 \mathrm{~m}$.............. } & an-0053 & 16 & $05: 35: 40$ & $6: 23: 20$ \\
\hline & an-0055 & 17 & 05:18:00 & $6: 33: 20$ \\
\hline & an- $0058^{\mathrm{a}}$ & 18 & $04: 59: 40$ & $6: 50: 10$ \\
\hline BAO $2.2 \mathrm{~m} \ldots \ldots \ldots \ldots \ldots$ & bao- $0037^{a}$ & 18 & $14: 47: 40$ & $3: 49: 20$ \\
\hline Mount Suhora $0.6 \mathrm{~m} . \ldots .$. & suh- $064^{\mathrm{a}}$ & 19 & $00: 05: 50$ & $3: 03: 50$ \\
\hline McDonald $2.1 \mathrm{~m}$........... & an-0060 & 19 & $05: 28: 20$ & $6: 18: 20$ \\
\hline
\end{tabular}

${ }^{a}$ Not used in the final analysis, because data were of low quality because of clouds, extinction, etc.

target for the WET campaign held in 1996 September. Here we report this endeavor to improve on previous attempts to decode this star. In $\S 2$, we describe the WET observations and their reduction. Section 3 outlines our results and their implications for previous mode identification and period spacing arguments. In $\S 4$, we discuss the physical properties of PG 0122 in light of the pulsation periods. Based on these results, $\S 5$ outlines future prospects for deducing the rate at

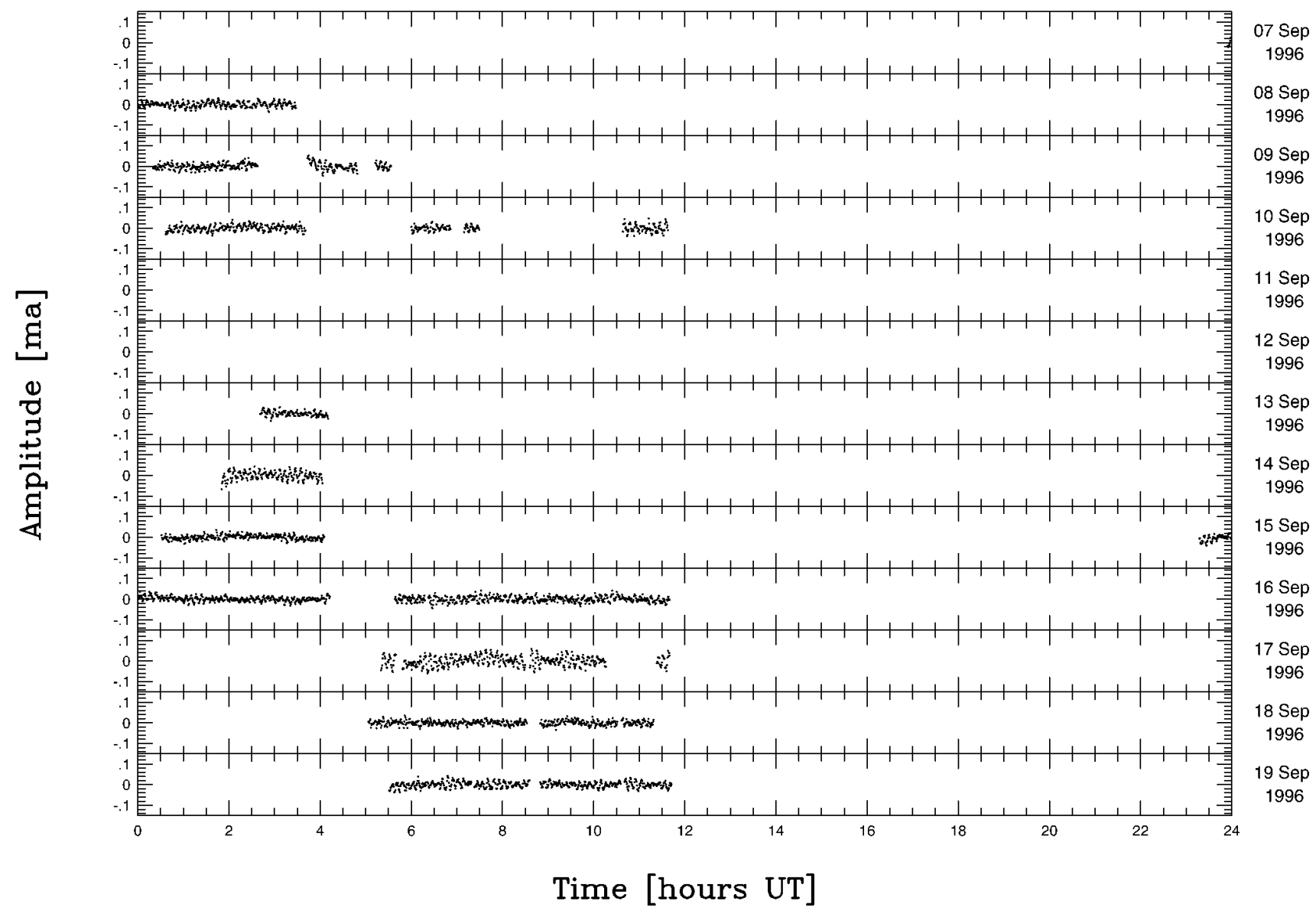

FIG. 1.-Light curve of PG 0122. The vertical axis has the units of modulation amplitude, ma ( $\equiv \Delta I / I)$. Each panel represents a single $24 \mathrm{hr} U T C$ day. 


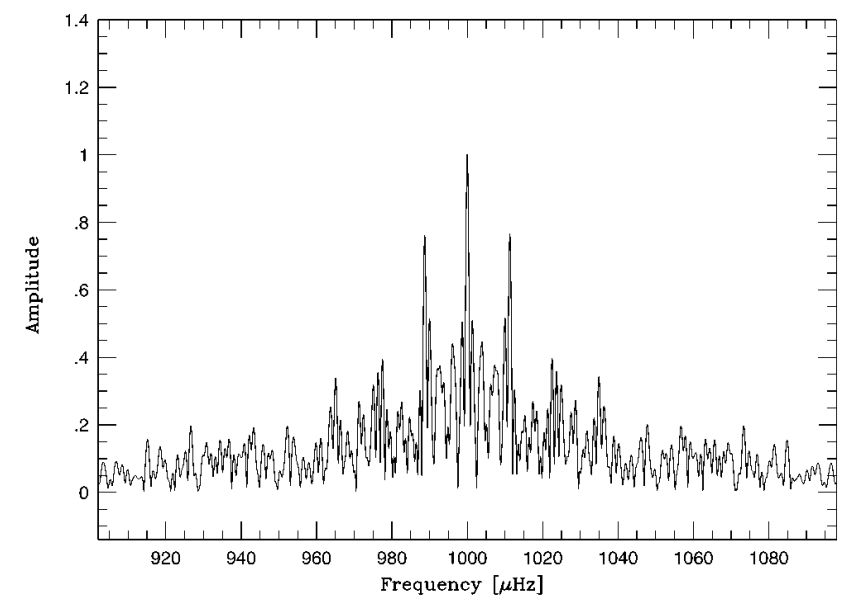

FIG. 2.- Spectral window of the WET run. The unusually large side lobes (for a WET run) are caused by the prominent daily gaps visible on the right-hand side of the light curve shown in Fig. 1.

which neutrinos escape the core of PG 0122 . We summarize and discuss our findings in $\S 6$.

\section{THE WET DATA SET}

With a battery of a dozen or so observatories from which to watch a single target, the Whole Earth Telescope generally enjoys advantages beyond the obvious ability to follow a star for longer than a few hours at a time. Chief of these is the weather: multiple redundancies at several longitudes give the WET better average weather conditions than can be claimed at all but the best single sites. In the past, this has resulted in relatively cloud-free data sets for every WET run. It did not in this one; hurricanes in two oceans contributed to the lowest overall duty cycle of any WET run held

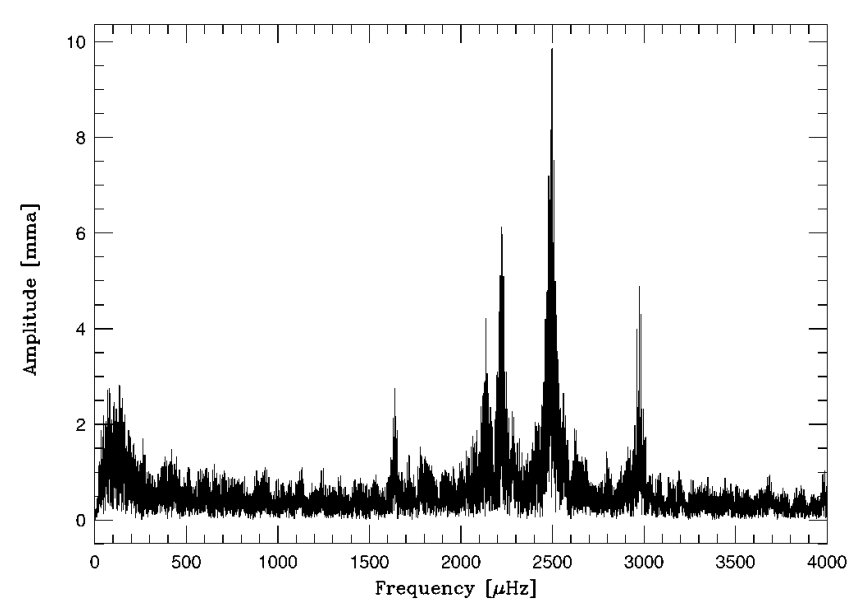

FIG. 3.-Amplitude spectrum of the WET data set, out to the highest frequency showing significant amplitude above noise. The amplitude is given in units of $\mathrm{ma}(\equiv \Delta I / I)$.

to date. Table 1 lists the observatories involved in data acquisition on PG 0122 and the resulting data runs. Only a subset of these data was appropriate for-and therefore used in - the final analysis, as noted in the table. Over a 13 day period in 1996 September, observers accumulated 13,757 integrations of $10 \mathrm{~s}$ each, an overall duty cycle of $14 \%$, far below the $50 \%-90 \%$ normally achieved by the WET.

Data acquisition and reduction followed the procedures outlined by Nather et al. (1990). The reduced data represent the fractional departure of the count rate from the mean at times referred to a common zero point of 2,450,334.495262 BJED, corresponding to the beginning of the first data set. All runs were combined into a single light curve, shown in Figure 1. Its most striking feature is the daily gap between

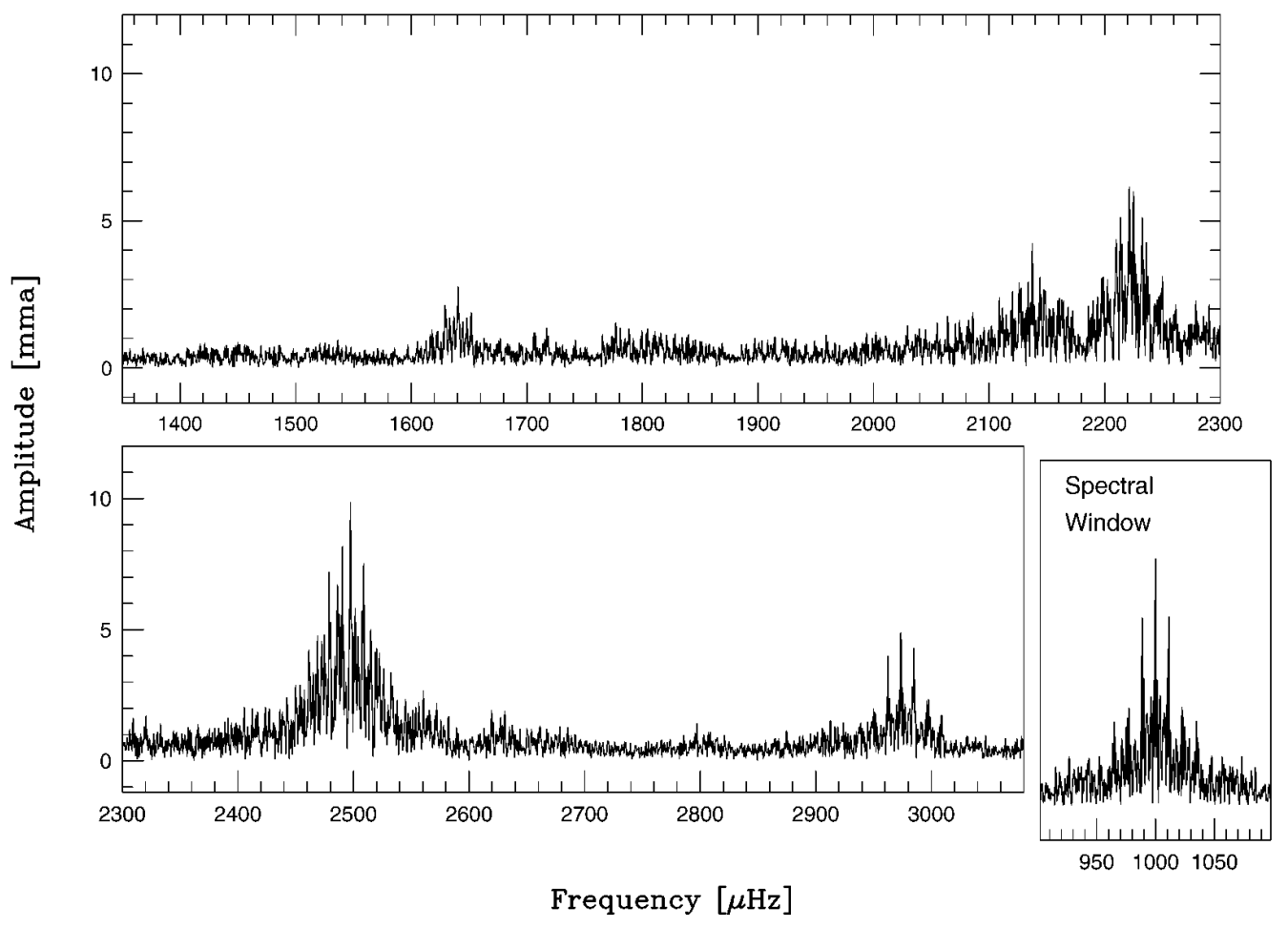

FIG. 4.- Region of the amplitude spectrum analyzed in this paper. For comparison, the spectral window shows the transform of a single noise-free sinusoid sampled at the same times as the data. 
12:00 and 23:00 UT created by poor weather conditions in China, a site for which we had no redundancy in this campaign. The general quality of the data coverage is exemplified by the spectral window, shown in Figure 2, which depicts the Fourier transform of a single noise-free sine function sampled at the same times as the WET data. The signature of the large daily gaps is evident in the notorious once-per-day aliasing side lobes that surround the central peak. Similar aliasing must affect each peak in the transform of the WET light curve.

The Fourier transform of the WET data set (Fig. 3) displays the modulation amplitude (in units of $\mathrm{mma}=\mathrm{ma} /$ 1000 , where $\mathrm{ma} \equiv \Delta I / I)$ of variations in the detected intensity $I$ at a given frequency. The low-frequency band of peaks below $100 \mu \mathrm{Hz}$ is an effect of slow extinction variations imperfectly removed during data reduction. Excluding these low frequencies, there are five obvious groups well above noise, all lying in the region between 1500 and 3000 $\mu \mathrm{Hz}$. Figure 4 shows this region on an expanded horizontal scale, with the spectral window included on the same scale for comparison. Six of the eight frequency groups found by OCKD are apparent here (the peaks near 1640, 2137, 2220, 2500, 2630, and $2980 \mu \mathrm{Hz}$ ), with relative amplitudes similar to those in the 1986 data. One additional peak near 2800 $\mu \mathrm{Hz}$, not present in either 1986 or 1990 , is possibly significant.

\section{RESULTS}

An original hope in assigning PG 0122 as the top-priority WET target during the 1996 fall campaign-besides the possibility of finding new modes - was to acquire a data set so complete as to allow unambiguous separation of closely spaced peaks in the Fourier transform. The rotational splitting claimed in previously published data could then be absolutely refuted or confirmed, with the inherent consequences for suggested mode identification and period spacing and hence for the calculated mass and luminosity of the star. Unfortunately, the complexity of our spectral window meant that we again had to resort to prewhitening to discern multiple closely spaced peaks, as was done with previous data sets.

\subsection{Frequency Identification}

Using the nonlinear least-squares procedure described in OCKD, we fitted the time-series data to obtain the frequency, amplitude, and phase of the five dominant modes in the light curve. After subtracting sinusoids with these parameters from the light curve, we recomputed the Fourier transform to see what was left. This process was then repeated, with an increased number of peaks included in each successive least-squares fit. In this way we found one additional peak (after initial prewhitening) near the largest mode in the $2220 \mu \mathrm{Hz}$ region, making this group a doublet. We also found two additional peaks near the largest mode in the transform at $2500 \mu \mathrm{Hz}$. The prewhitening sequence is shown in Figure 5 in the $2500 \mu \mathrm{Hz}$ region. The figure indicates the peaks that were identified and the results of each subsequent subtraction. Figure 6 compares the WET transform to that of a noise-free light curve constructed using the three peaks identified in Figure 5. The three peaks form a triplet of equal frequency spacing, with $\langle\delta v\rangle=3.581 \pm 0.006 \mu \mathrm{Hz}$. This is an important confirmation of the results of OCKD, who identified precisely these three frequencies, though less conclusively. The triplet

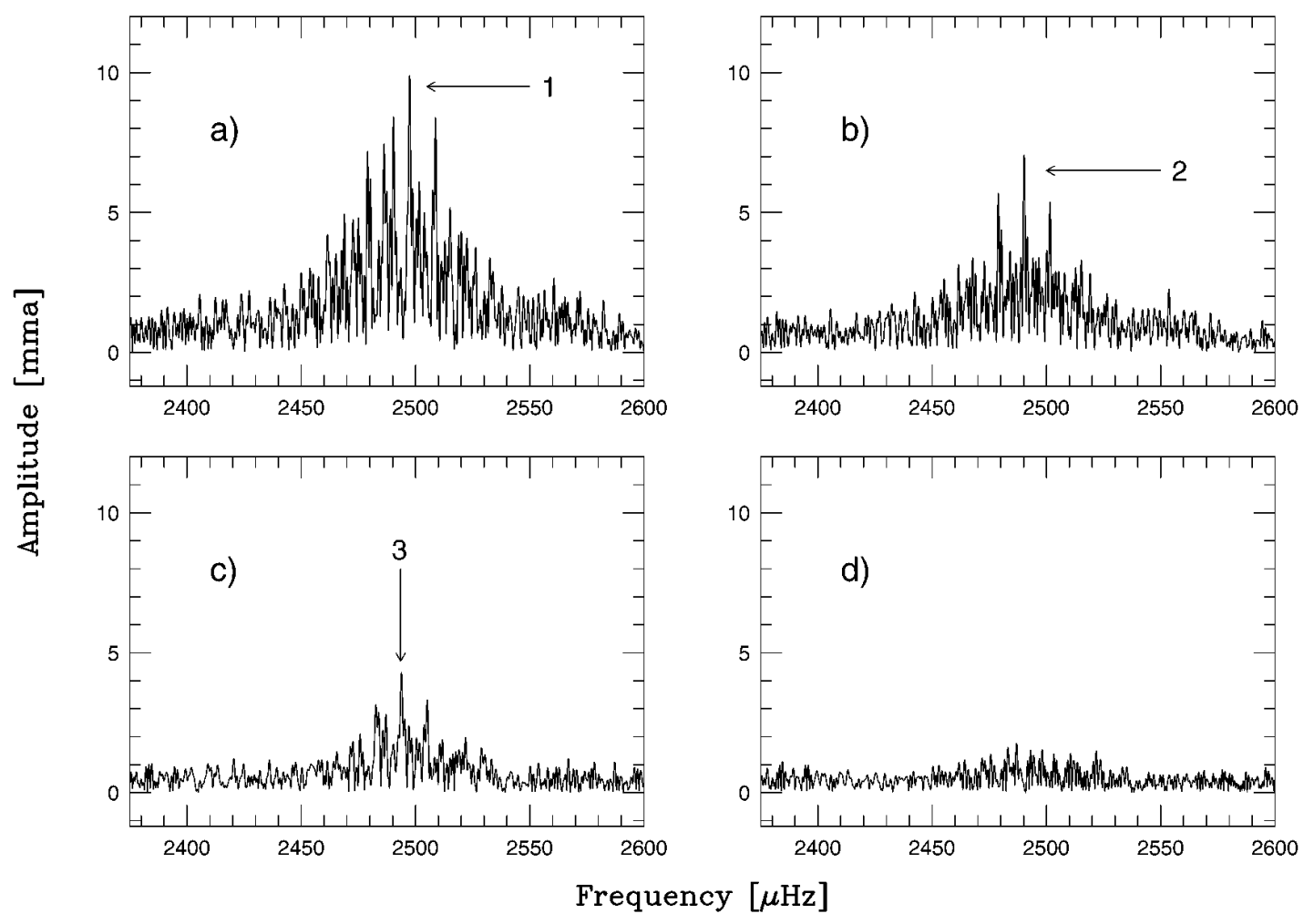

FIG. 5.-Prewhitening sequence for the region of largest amplitude in the spectrum; $(a)$ the original spectrum; $(b)$, (c), and (d) show the effects of subtraction in the time domain of one, two, and three sine waves (with periods corresponding to the peaks labeled 1-3), respectively, from the light curve. Frequencies, amplitudes, and phases for each subtracted mode were obtained by simultaneous least-squares fitting to the original light curve. 
TABLE 2

Periodicities of PG 0122 From the WET Data SeT

\begin{tabular}{ccccccccc}
\hline \hline $\begin{array}{c}\text { Period } \\
(\mathrm{s})\end{array}$ & $\begin{array}{c}\text { Frequency } \\
(\mu \mathrm{Hz})\end{array}$ & $\begin{array}{c}\sigma_{f} \\
(\mu \mathrm{Hz})\end{array}$ & $\begin{array}{c}\text { Amplitude } \\
(\mathrm{ma})\end{array}$ & $\begin{array}{c}\sigma_{A} \\
(\mathrm{ma})\end{array}$ & $\ell$ & $m$ & $\begin{array}{c}\delta v \\
(\mu \mathrm{Hz})\end{array}$ & $\begin{array}{c}\sigma_{\delta v} \\
(\mu \mathrm{Hz})\end{array}$ \\
\hline $609.5729 \ldots \ldots$ & 1640.493 & 0.004 & 2.7 & 0.2 & $1^{\mathrm{a}}$ & $-1^{\mathrm{a}}$ & $7.9^{\mathrm{a}}(2 \times 3.85)$ & 0.2 \\
$467.8656 \ldots \ldots$ & 2137.366 & 0.003 & 4.5 & 0.2 & $\ldots$ & $\ldots$ & $\ldots$ & $\ldots$ \\
$450.1753 \ldots \ldots$ & 2221.357 & 0.002 & 5.3 & 0.3 & 1 & $+1^{\mathrm{b}}$ & 3.332 & 0.003 \\
$449.5010 \ldots \ldots$ & 2224.689 & 0.002 & 5.1 & 0.3 & 1 & $0^{\mathrm{b}}$ & $\ldots$ & $\ldots$ \\
$401.5558 \ldots \ldots$ & 2490.314 & 0.002 & 8.1 & 0.3 & 1 & +1 & 3.585 & 0.004 \\
$400.9785 \ldots \ldots$ & 2493.899 & 0.003 & 4.2 & 0.4 & 1 & 0 & 3.577 & 0.004 \\
$400.4042 \ldots \ldots$ & 2497.476 & 0.002 & 8.5 & 0.3 & 1 & -1 & $\ldots$ & $\ldots$ \\
$380.1111 \ldots \ldots$ & 2630.810 & 0.004 & 2.6 & 0.2 & $1^{\mathrm{a}}$ & $+1,0^{\mathrm{a}}$ & $3.3^{\mathrm{a}}$ & 0.3 \\
$336.2804 \ldots \ldots$ & 2973.709 & 0.002 & 5.4 & 0.2 & $1^{\mathrm{ab}}$ & $-1^{\mathrm{ab}}$ & $3.70^{\mathrm{ab}}$ & 0.2 \\
\hline
\end{tabular}

NoTES.- Numbers in parentheses show the mean consecutive $m$ splitting if the observed doublet is assumed to represent $m= \pm 1, \ell=1$. A comma separates possible $m$ identifications where constraint to a single value was not possible. Frequency and amplitude errors derive from a formal least-squares analysis of the data. The amplitude units are modulation amplitude ma $(\equiv \Delta I / I)$.

${ }^{a}$ Based on the frequency of a nearby peak in data from O'Brien et al. 1996.

${ }^{b}$ Based on the frequencies of nearby peaks in the data of Vauclair et al. 1995.

structure suggests an $\ell=1$ mode; the period spacing (see $\S 3.2)$ is consistent with this identification for reasonable white dwarf masses.

In all, we found nine individual periodicities in the WET data, making up four singlets, a doublet, and a triplet. Table 2 lists the modes and their identification (values of $\ell$ and $m$ ). In some cases, mode identification was aided by compari-

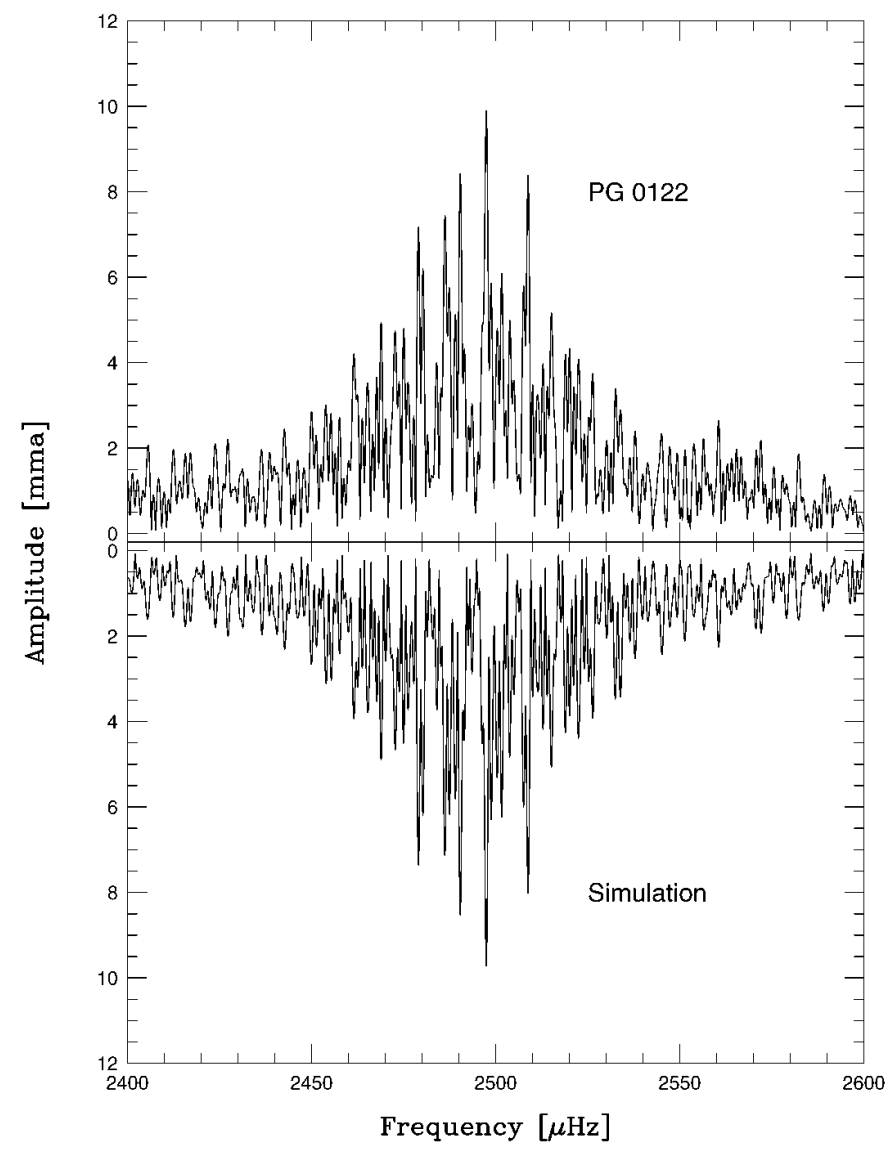

FIG. 6.-Comparison of a portion of the amplitude spectrum of PG 0122 to the spectrum of a (noise-free) light curve constructed using the three peaks identified by prewhitening. The transform of the simulated light curve has been inverted in order to aid comparison of the two spectra by eye. son with the peak lists of OCKD and VPG, reproduced here in Tables 3 and 4, respectively. Thus, based on the observed frequency splitting, five of the six groups found in the WET data set belong to $\ell=1$ modes. The average consecutive $m$ frequency splitting for all the $\ell=1$ modes is $\langle\delta v\rangle=3.5 \pm 0.2 \mu \mathrm{Hz}$, which from equations (1) and (2) of Kawaler et al. (1995; also see Brickhill 1975; Winget et al. 1991) implies a rotation rate of $1.6 \pm 0.1$ days.

The final peak, at $2137 \mu \mathrm{Hz}$, lies at the position of an alias of a peak previously identified by OCKD. This calls into question their identification of this mode, based on frequency splitting arguments, as $\ell=2$. At present, the value of $\ell$ for this peak remains unconstrained by the data.

Our inability to resolve with this data set the identity of the $2137 \mu \mathrm{Hz}$ mode is disappointing, for this region has been a source of particular difficulty. While OCKD tentatively identified it as an $\ell=2$ mode based on the larger splitting found in what was then a doublet, VPG suggested the possibility of an accidental coincidence of both $\ell=1$ and $\ell=2$ modes, though this idea was not based on observation of both $\ell=1$ and $\ell=2$ splitting within the mode.

TABLE 3

PeRIODICITIES OF PG 0122

\begin{tabular}{ccccccc}
\hline \hline $\begin{array}{c}\text { Period } \\
(\mathrm{s})\end{array}$ & $\begin{array}{c}\text { Frequency } \\
(\mu \mathrm{Hz})\end{array}$ & $\begin{array}{c}\sigma_{f} \\
(\mu \mathrm{Hz})\end{array}$ & $\begin{array}{c}\text { Amplitude } \\
(\mathrm{ma})\end{array}$ & $\begin{array}{c}\sigma_{A} \\
(\mathrm{ma})\end{array}$ & $\ell$ & $m$ \\
\hline $612.44 \ldots \ldots$ & 1632.8 & 0.2 & 2.6 & 0.3 & $1^{\mathrm{a}}$ & $+1^{\mathrm{a}}$ \\
$570.00 \ldots \ldots$ & 1754.4 & 0.2 & 2.5 & 0.3 & $?$ & $?$ \\
$466.37 \ldots \ldots$ & 2144.2 & 0.2 & 3.3 & 0.4 & $?$ & $?$ \\
$465.20 \ldots \ldots$ & 2149.6 & 0.2 & 2.3 & 0.4 & $?$ & $?$ \\
$450.25 \ldots \ldots$ & 2221.0 & 0.1 & 6.3 & 0.4 & $1^{\text {ab }}$ & $-1^{\text {ab }}$ \\
$401.61 \ldots \ldots$. & 2490.0 & 0.1 & 7.3 & 0.4 & 1 & +1 \\
$401.03 \ldots \ldots$. & 2493.6 & 0.2 & 3.0 & 0.4 & 1 & 0 \\
$400.50 \ldots \ldots$ & 2496.9 & 0.1 & 12.3 & 0.4 & 1 & -1 \\
$380.08 \ldots \ldots$ & 2631.0 & 0.2 & 1.9 & 0.4 & 1 & $+1,0$ \\
$379.61 \ldots \ldots$ & 2634.3 & 0.2 & 2.1 & 0.4 & 1 & $0,-1$ \\
$337.13 \ldots \ldots$ & 2966.2 & 0.2 & 3.1 & 0.4 & 1 & +1 \\
$336.29 \ldots \ldots$ & 2973.6 & 0.1 & 5.1 & 0.4 & 1 & -1 \\
\hline
\end{tabular}

Notes. - Data are from O'Brien et al. 1996, identified in single-site data taken in 1986. Frequency and amplitude errors derive from a formal least-squares analysis of the data. The amplitude units are modulation amplitude ma $(\equiv \Delta I / I)$.

${ }^{a}$ Based on the frequency of a nearby peak in the WET data set.

b Based on the frequencies of nearby peaks in the data of Vauclair et al. 1995. 
TABLE 4

PeRIODICITIES OF PG 0122

\begin{tabular}{cccrr}
\hline \hline $\begin{array}{c}\text { Period } \\
(\mathrm{s})\end{array}$ & $\begin{array}{c}\text { Frequency } \\
(\mu \mathrm{Hz})\end{array}$ & $\begin{array}{c}\text { Amplitude } \\
(\mathrm{ma})\end{array}$ & $\ell$ & \multicolumn{1}{c}{$m$} \\
\hline $450.13 \ldots \ldots$ & 2221.6 & 1.1 & 1 & +1 \\
$449.44 \ldots \ldots$. & 2225.0 & 1.5 & 1 & 0 \\
$448.89 \ldots \ldots$ & 2227.7 & 1.2 & 1 & -1 \\
$401.06 \ldots \ldots$. & 2493.4 & 1.6 & $?$ & $?$ \\
$400.90 \ldots \ldots$ & 2494.4 & 1.8 & 1 & 0 \\
$400.42 \ldots \ldots$. & 2497.4 & 4.2 & 1 & -1 \\
$336.68 \ldots \ldots$ & 2970.2 & 0.7 & 1 & 0 \\
$336.28 \ldots \ldots$ & 2973.7 & 1.8 & 1 & -1 \\
\hline
\end{tabular}

Notes.- Data are from Vauclair et al. 1995, identified in bilongitudinal data taken in 1990. The amplitudes, given by Vauclair et al. in units of mmag, have been converted to modulation amplitude ma $(\equiv \Delta I / I)$.

Identification of the value of $\ell$ for this mode is important because of its close proximity in period to the mode at 2220 $\mu \mathrm{Hz}$. This proximity led VPG to suggest an $\ell=1$ period spacing of $\sim 16 \mathrm{~s}$, while OCKD claim a period spacing of $21.2 \mathrm{~s}$. The possibility of observing several components of the $2137 \mu \mathrm{Hz}$ mode and thereby conclusively deducing its order $\ell$ was thus an exciting prospect. Unfortunately, after prewhitening of the WET light curve at the single observed frequency, there remained only an indistinguishable jumble in the transform (Fig. 7), a possible effect of aliasing interference between two or more real periodicities of similar small amplitude. Until we obtain a more complete record of the light curve of PG 0122, the $2137 \mu \mathrm{Hz}$ mode will remain a puzzle.

\subsection{Period Spacings}

Each of the three pulsating pre-white dwarfs studied with multisite data so far-PG 1159, PG 2131, and NGC 1501shows a pattern of $\ell=1$ modes spaced at multiples of a fundamental period interval. This interval is determined by the set of modes available for pulsation; not all theoretically available modes are excited, leaving gaps of one or more "missing" periods in a chain mostly consecutive in radial index $n$. The fundamental period spacing is the most important parameter measured in the pulsation spectrum of a white dwarf star, as it is determined almost exclusively by two parameters, the stellar mass and luminosity. The first step in deducing this spacing is identification of the order $\ell$ of each of the modes in the pulsation spectrum, a process in

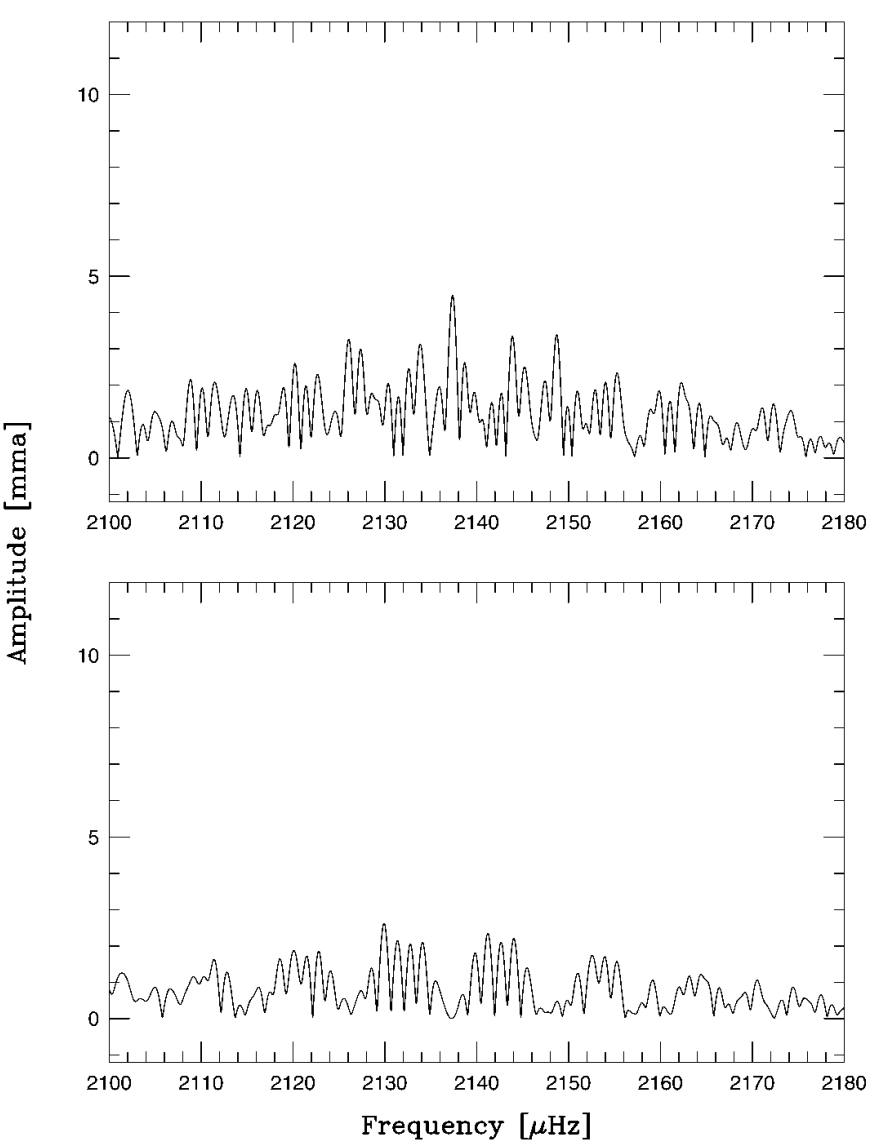

Fig. 7.-Transform before (upper panel) and after (lower panel) prewhitening of the WET light curve by the $2137 \mu \mathrm{Hz}$ peak. The unusually high "noise band" suggests the possible existence of additional modes in this controversial region of the transform, but, if so, these remain indecipherable in the WET data set.

this case outlined in the previous section. As mentioned above, VPG and OCKD have already attempted to explain the period spectrum of PG 0122 with different values of the primary spacing. We can now use the observed periods to attempt to decide between one or the other of these hypotheses.

Table 5 compares the observed pattern of $\ell=1$ modes to periods predicted by strict patterns of $21.10 \mathrm{~s}$ and $16.24 \mathrm{~s}$ spacing. These values were obtained by taking the likely

TABLE 5

Comparison of Period Spectrum with Patterns of Strict 21.10 s and 16.24 s Spacing BETWEEN MODES OF CONSECUTIVE RADIAL INDEX $n$

\begin{tabular}{|c|c|c|c|c|c|c|}
\hline \multirow[b]{2}{*}{$\begin{array}{c}\Pi_{\text {observed }}(m=0) \\
(\mathrm{s})\end{array}$} & \multicolumn{3}{|c|}{$\Delta \Pi=21.10 \mathrm{~s}$} & \multicolumn{3}{|c|}{$\Delta \Pi=16.24 \mathrm{~s}$} \\
\hline & $\begin{array}{c}\Pi_{\text {pred }} \\
(\mathrm{s})\end{array}$ & $n-n_{0}$ & $\begin{array}{c}\Pi_{\mathrm{obs}}-\Pi_{\text {pred }} \\
\text { (s) }\end{array}$ & $\begin{array}{c}\Pi_{\text {pred }} \\
(\mathrm{s})\end{array}$ & $n-n_{0}$ & $\begin{array}{l}\Pi_{\mathrm{obs}}-\Pi_{\text {pred }} \\
\text { (s) }\end{array}$ \\
\hline 611.0*. & 612.0 & +10 & -1.0 & 612.1 & +13 & -1.1 \\
\hline $570.0^{\mathrm{a}}\left(\begin{array}{c}+1.2 \\
-1.3\end{array}\right) \ldots \ldots$ & 569.8 & +8 & $+0.2\left(\begin{array}{c}+1.2 \\
-1.3\end{array}\right)$ & 563.4 & +10 & $+6.6\left(\begin{array}{l}+1.2 \\
-1.3\end{array}\right)$ \\
\hline $449.5 \ldots \ldots \ldots \ldots \ldots$ & 443.2 & +2 & +6.3 & 449.7 & +3 & -0.2 \\
\hline $401.0 \ldots \ldots \ldots \ldots$ & 401.0 & 0 & & 401.0 & 0 & \\
\hline $379.6,380.1 \ldots \ldots$ & 379.9 & -1 & $-0.3,+0.2$ & 384.8 & -1 & $-5.2,-4.7$ \\
\hline $336.7 \ldots \ldots \ldots \ldots$ & 337.7 & -3 & -1.0 & 336.0 & -4 & -0.7 \\
\hline
\end{tabular}

Notes.-Period spectrum at $(\ell=1, m=0)$. An asterisk indicates that the period represents the calculated center of a doublet splitting. Numbers in parentheses show the effects of assuming a value of $m$ other than $m=0$ for the identified peak.

a Mode identified by O'Brien et al. 1996. Not observed in the WET data set. 
value of $\Delta n\left(=n-n_{0}\right)$ for each mode based only on approximate spacings of $21 \mathrm{~s}$ and $16 \mathrm{~s}$, and then, for the two resulting sets of $\Delta n$ (one for each estimate of $\Delta \Pi$ ), fitting a line to the equation

$$
\Pi_{n}=\Delta \Pi \times \Delta n+\Pi_{n_{0}},
$$

where the $\Pi_{n}$ are the observed periods and both $\Delta \Pi$ and $\Pi_{n_{0}}$ are allowed to vary. $\Delta \Pi=21.10 \mathrm{~s}$ and $16.24 \mathrm{~s}$ represent the two best fits (in a least-squares sense) to the observed periods, given the assumed values of $\Delta n$ listed in Table 3 . Other values of $\Delta \Pi$, using yet other assumed sets of $\Delta n$, can be chosen to fit the data, but none leads to sensible estimates for the mass of PG 0122.

The smallest observed spacing in the period spectrum between modes confidently identified as $\ell=1$ is $\sim 21 \mathrm{~s}$, between the modes at $401 \mathrm{~s}$ and $380 \mathrm{~s}(2500 \mu \mathrm{Hz}$ and 2630 $\mu \mathrm{Hz}$ ), and this formed the seed of the original suggestion of $21 \mathrm{~s}$ as the average period spacing in the spectrum of PG 0122. The $380 \mathrm{~s}$ mode is also one of two (the other being at $570 \mathrm{~s}$ ) that the proposed $16.2 \mathrm{~s}$ pattern misses rather significantly. The $21.1 \mathrm{~s}$ pattern, on the other hand, fails at about the same level to explain the mode at $450 \mathrm{~s}(2225 \mu \mathrm{Hz})$. All of these "misses" could result from effects of mode trapping, so that both patterns account for the data almost equally well, with $\Delta \Pi=21.1$ s somewhat preferred. We previously mentioned an additional peak tantalizingly close to $1 \sigma$ above the local noise band at $2800 \mu \mathrm{Hz}$ (357.1 s). Though it is not by itself strictly significant, if real, this peak would fit the $21.1 \mathrm{~s}$ pattern precisely - completing a chain of four consecutive modes - and would in addition allow us to rule out a $16.2 \mathrm{~s}$ pattern with high confidence. While this peak is largest in the transform between the $\ell=1$ groups near $336 \mathrm{~s}$ and $380 \mathrm{~s}$, we cannot claim with certainty that it is a real pulsation mode if considered entirely on its own merits. We can say only that while $\Delta \Pi \cong 21 \mathrm{~s}$ is the most probable solution to the pulsation spectrum of PG 0122, $\Delta \Pi \cong 16 \mathrm{~s}$ cannot, at this point, be ruled out conclusively.

\section{THE MASS AND LUMINOSITY OF PG 0122}

Given the two measured quantities $\Delta \Pi$ (from the pulsation data) and $T_{\text {eff }}$ (from spectroscopy), stellar models can be used to place limits on the mass and luminosity of a pulsating pre-white dwarf star. Dreizler et al. (1995) find a $T_{\text {eff }}$ for PG 0122 of $75,000 \pm 5000 \mathrm{~K}$. For pre-white dwarf models with a given mass, the period spacing increases with decreasing effective temperature. In addition, for models with a given effective temperature, period spacing increases with decreasing mass. Thus for PG 0122 to maintain a period spacing (21 s) similar to that of PG 1159-which is far hotter at $T_{\text {eff }}=140,000 \mathrm{~K}$-it must be more massive. If the period spacing is even shorter (16 s), it must be more massive still. Based on stellar models developed by Dehner $\&$ Kawaler (1995), the two values of $\Delta \Pi$ discussed above imply a mass for PG 0122 of either $0.69 \pm 0.03 M_{\odot}$ (for $\Delta \Pi=21.1 \mathrm{~s})$ or $1.00 \pm 0.08 M_{\odot}$ (for $\left.\Delta \Pi=16.2 \mathrm{~s}\right){ }^{22}$ The luminosities corresponding to these two mass ranges are $5.2 \pm 2.1 L_{\odot}$ and $1.5 \pm 0.8 L_{\odot}$, respectively. Either solution makes PG 0122 the most massive and least intrinsically luminous GW Virginis star known. This result has impor-

\footnotetext{
${ }^{22}$ If these modes are $\ell=2$, the implied mass is less than $0.4 M_{\odot}$. This is below the minimum mass for a $\mathrm{C} / \mathrm{O}$ white dwarf and supports our identification $\ell=1$.
}

tant consequences for the character of the cooling mechanisms responsible for the star's continuing evolution, as we discuss in the next section.

\section{NEUTRINOS AND $d \Pi / d t$}

PG 0122 is, as we have shown, a very stable pulsator: over the past decade, it has shown a very consistent pulsation spectrum, with the large-amplitude modes present at the same frequencies during each of the three intensive observing seasons in 1986, 1990, and 1996. The amplitudes of each of the dominant modes remained approximately constant as well. ${ }^{23}$ Therefore, PG 0122 is an excellent candidate for measurement of the rate of secular period change caused by the evolutionary cooling of its interior.

Measurements of secular period change $(d \Pi / d t)$ in white dwarfs have been attempted by a number of investigations, with either measurements made or tight upper limits set for the GW Virginis stars PG 1159 (Winget et al. 1985, 1991) and G117-B15A (Kepler et al. 1995). In order to measure $d \Pi / d t$ with confidence, the star must have a stable and fully resolved pulsation with decent phase measurements from season to season. For the GW Virginis stars, measurement of $d \Pi / d t$ is important for astrophysical reasons in that it helps constrain the possible progenitor models. More interestingly, since neutrino emission plays an important role in the rate of cooling of hot white dwarfs, measurement of this cooling rate through $d \Pi / d t$ allows a measurement of the rate of neutrino production in dense plasma. The rates used in the models are computed using standard electroweak theory in a region of parameter space that has not been tested experimentally; thus agreement (or disagreement) between theory and observation is an interesting astrophysical test of the standard model of particle physics.

Kawaler et al. (1985) and Kawaler \& Bradley (1994) present predicted values of $d \Pi / d t$ for models relevant to GW Virginis stars; the only observed value of $d \Pi / d t$, that for PG 1159 itself, is consistent with these models. However, as Kawaler \& Bradley (1994) demonstrated, for a star as hot as PG $1159, d \Pi / d t$ is strongly affected by mode trapping. Kawaler \& Bradley (1994) show that, in general, $d \Pi / d t$ should be positive, reflecting the overall cooling of the model (Winget, Hansen, and Van Horn 1983). However, trapped modes are concentrated in the outer layers, within which contraction dominates cooling; therefore, trapped modes can show periods that decrease with time. Thus, mode trapping can complicate the interpretation of measured period changes in hot GW Virginis stars.

As will be shown in a subsequent paper (O'Brien \& Kawaler 1998), period changes in models with lower effective temperatures are dominated more and more by a single physical process. For cooler GW Virginis stars, neutrinos provide an increasing contribution to total luminosity. Also, the neutrino contribution to cooling increases with increasing mass. Figure 8 shows these effects for two models used in the analysis by OCKD. The two lines show the ratio of the neutrino energy loss rate $\left(L_{v}\right)$ to the photon luminosity $\left(L_{\gamma}\right)$ for models with masses of $0.66 M_{\odot}$ (relevant for

\footnotetext{
${ }^{23}$ The amplitudes in 1990, as reported by VPG, average 3.6 times smaller than found in both 1986 and 1996. However, examination of the original data from 1990, kindly provided by Al Grauer (1997, private communication), shows that the amplitudes in 1990 were essentially the same as in 1986 and 1996. The lower amplitudes reported by VPG appear to be in error.
} 


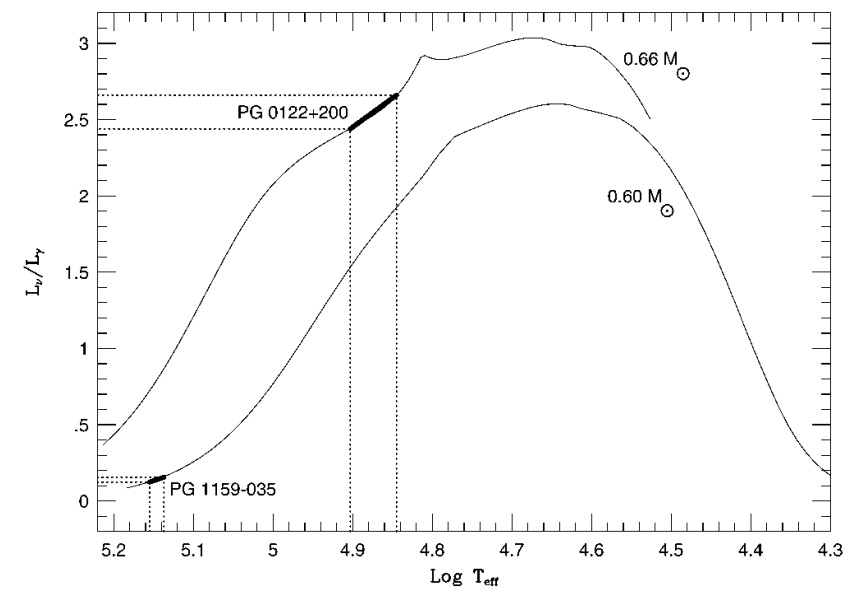

Fig. 8.-Ratio of the neutrino luminosity to the photon luminosity as a function of $T_{\text {eff }}$ for two stellar models of different mass, $M_{*}=0.60 M_{\odot}$ and $M_{*}=0.66 M_{\odot}$.

PG 0122) and $0.60 M_{\odot}$ (appropriate for PG 1159). At large effective temperatures, the neutrino contribution is very small; PG 1159 , at $140,000 \mathrm{~K}$, is cooled by neutrinos at the $10 \%$ level; when it cools to $80,000 \mathrm{~K}$, the neutrino contribution will be more than double that of the photon luminosity. It is also clear in Figure 8 that the neutrino contribution to energy loss is always larger in the more massive model. At $75,000 \mathrm{~K}$ in the $0.66 M_{\odot}$ model-again, relevant for PG 0122 - the neutrino luminosity is nearly 3 times that of the photon luminosity.

In addition to the physics governing neutrino production, PG 0122 is an ideal target for measuring neutrino production rates because of the decreasing influence of mode trapping on interpretation of $d \Pi / d t$ with decreasing $T_{\text {eff }}$. As GW Virginis stars cool, the surface contraction rate decreases relative to the cooling rate of the interior. So, while mode trapping can still influence the pulsation period distribution, the rates of period change become more similar from mode to mode in cooler GW Virginis stars. Kawaler \& Bradley (1994) found that the sign of $d \Pi / d t$ could be different for different modes in hot GW Virginis models; by the time those same models cool to temperatures relevant for PG 0122, the period change rates are all positive. Figure 9 shows the rate of period change for modes in a model with $0.66 M_{\odot}$ and $T_{\text {eff }}=75,000 \mathrm{~K}$. Com-

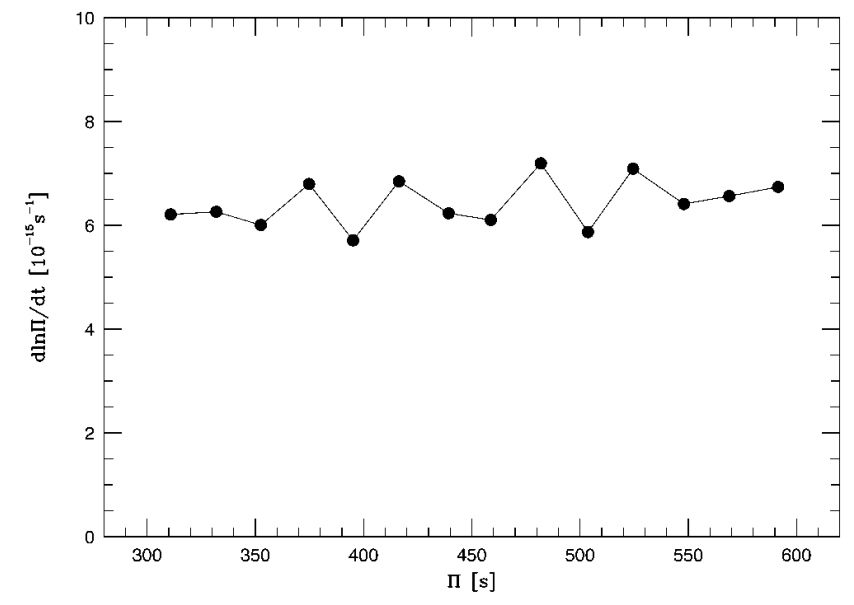

Fig. 9.-Period derivative for 14 consecutive pulsation modes in a stellar model with $T_{\text {eff }}=75,000 \mathrm{~K}$ and $M=0.66 M_{\odot}$. parison of this figure with the figures shown in Kawaler \& Bradley (1994) shows a third reason why measurement of $d \Pi / d t$ in PG 0122 will provide a much tighter constraint on the rate of evolution of pre-white dwarf stars.

We can use Figure 9 to estimate the value of $d \Pi / d t$ for PG 0122 and estimate the prospects for measuring this value. The value of $d \Pi / d t$ for the $401 \mathrm{~s}$ mode should be about $3 \times 10^{-12} \mathrm{~s} \mathrm{~s}^{-1}$. To measure $d \Pi / d t$ in white dwarf stars, the favored technique is to detect the cumulative phase drift in a mode with changing period. This is accomplished by comparing the observed times of maxima $(O)$ in the light curve to the calculated times of maxima $(C)$ based on an assumption of constant period. The resulting plot of $O-C$ shows the phase drift associated with a changing period. A constant rate of period change, $d \Pi / d t$, enters as a quadratic term in time:

$$
(O-C) \approx \frac{1}{2} \frac{1}{\Pi_{t_{0}}} \frac{d \Pi}{d t}\left(t-t_{0}\right)^{2} \mathrm{~s},
$$

where $\Pi_{t_{0}}$ is the period at time $t_{0}$ (see, e.g., Winget et al. 1985, 1991; Kepler et al. 1995). With this rate of period change, the period would change by about $0.001 \mathrm{~s}$ in $10 \mathrm{yr}$; this is smaller than the period uncertainty for a run length of several months (assuming a frequency precision of $1 / 10$ the run length). However, the accumulated phase advance over a 10 yr period should be nearly two full cycles.

Using the periods alone from the 1986, 1990, and 1996 data, we can attempt to calculate $d \Pi / d t$ directly. From the best least-squares periods from 1996 and 1986, we calculate a period change of $-0.10 \pm 0.02 \mathrm{~s}$, implying $d \Pi /$ $d t=-3 \times 10^{-10} \mathrm{~s} \mathrm{~s} \mathrm{~s}^{-1}$, about 100 times larger in magnitude - and different in sign - than theory expects. However, this calculation is based on the formal errors from a least-squares fitting to the observed periods, and the formal least-squares error generally underestimates the true error by an order of magnitude. In practice, the data currently available allow an upper limit to be set on $d \Pi / d t$ for PG 0122 of $6 \times 10^{-10} \mathrm{~s} \mathrm{~s}^{-1}$. In view of the importance of measuring $d \Pi / d t$ for this star (and for the other known cool GW Virginis stars PG 2131 and PG 1707), we must continue analysis of archival data and mount observing campaigns in the near future to monitor PG 0122. With frequent observation, an accumulated phase advance of half a cycle could be used to determine $d \Pi / d t$ in 3 yr. The data presented here provide a key anchor for this analysis.

\section{SUMMARY AND CONCLUSIONS}

Time-series photometry of the GW Virginis star PG 0122 obtained with the Whole Earth Telescope confirmed important results tentatively suggested in previous studies. The $\ell=1$ frequency splitting confirmed by this analysis sets the rotation rate at $1.6 \pm 0.1$ days. In addition, the previously suggested period spacing of $21 \mathrm{~s}$ is placed on firmer ground, though a pattern based on $16 \mathrm{~s}$ cannot be ruled out. PG 0122 - already known from spectroscopy to be the coolest GW Virginis star at 75,000 $\mathrm{K}$-is the most massive star in its class, with a mass of $0.69 \pm 0.03 M_{\odot}$ (if $\Delta \Pi \cong 21 \mathrm{~s}$ ).

Its relatively low temperature and high mass imply that PG 0122 cools primarily through neutrino emission, in contrast to most white dwarfs and pre-white dwarfs, which cool through normal photon luminosity. This suggests that PG 0122 (and cool GW Virginis stars in general) might be used to test predictions of standard lepton theory. To do 
this, the cooling rate must be deduced via measurement of the rate of period change, a goal that can be achieved in several years with frequent observation.

A PG 0122 period spacing near $21 \mathrm{~s}$ continues a trend that is slowly emerging among variable pre-white dwarf stars. Four of these, PG 1159, PG 2131, PG 0122, and the central star of the planetary nebula NGC 1501, have so far yielded to asteroseismological scrutiny. All show patterns of equal period spacing very close to $21 \mathrm{~s}$. Period spacing in pre-white dwarf models is primarily determined by two parameters, stellar mass and luminosity. From spectroscopic measurements of $T_{\text {eff }}$ and $\log g$, we know that these four stars are spread over 3 orders of magnitude in total luminosity. Thus, the sameness of $\Delta P$ from star to star is evidence for a wide spread in stellar mass among pulsating pre-white dwarfs. More specifically, an almost uniform $\Delta P$ within the instability strip implies that the lower the luminosity of a pulsating pre-white dwarf star, the larger is its mass.

The cause of this mass-luminosity relationship is a mystery. We know of no obvious reason for a correlation between mass and luminosity, though if confirmed it could provide clues toward the solution of another mystery: why pre-white dwarfs pulsate at all. For instance, their smaller masses imply that the more luminous stars PG 1159 and NGC 1501 formed from interstellar clouds up to several billion years earlier than the more massive PG 2131 and PG 0122. This in turn implies a metallicity gradient along the instability strip. Perhaps this is an important factor in understanding the origin of the pulsational instability. The answer to these questions lies in continued, persistent, relentless observation, coupled with insightful theory. We need to solve the pulsation spectra of several more individ- ual stars. In particular, the dogged persistence of the $21 \mathrm{~s}$ spacing is so intriguing that it begs for swift verification or refutation through the characterization of additional prewhite dwarf light curves.

We wish to thank $\mathrm{Al}$ Grauer for providing us with the archival data from 1990. We also thank Darragh O'Donoghue and Lee Anne Willson for enlightening discussions and helpful comments. Funding agencies around the globe made this research possible, and they have our gratitude. Specifically, M. S. O'B. enjoys support as a GAANN fellow through grant P200A10522 from the Department of Education to Iowa State University. S. D. K. acknowledges support from an NSF Young Investigator award to Iowa State University (grant AST-9257049). J. C. C. acknowledges support from NASA through grant HF-01041.01-93A from the Space Telescope Science Institute, which is operated by the Association of Universities for Research in Astronomy under NASA contract NAS 526555, and from the Sherman Fairchild Foundation. M. A. W. received partial support through NSF grant AST 9217988 to the Florida Institute of Technology and also from the NASA Astrophysics Theory Program (grant NAG 5-3103). The Texas WET group acknowledges support from NSF grant AST 93-15461. The Polish WET group is supported by the Polish Committee for Scientific Research through grant 2-P03D-015-08. The International Institute of Theoretical and Applied Physics (IITAP) at Iowa State University supported travel, meals, and lodging for visiting control center staff (M. S. O’B., S. J. K., J. L. P., T. K. W., M. A. W., and J. K.) through contract CP/RP 206.590.6 from the United Nations Educational, Scientific, and Cultural Organization (UNESCO).
Appleton, P. N., Kawaler, S. D., \& Eitter, J. J. 1993, AJ, 106, 1973

Bond, H. E., \& Grauer, A. D. 1987, ApJ, 321, L123

Bond, H. E., et al. 1996, AJ, 112, 2699

Brickhill, A. J. 1975, MNRAS, 170, 405

Dehner, B., \& Kawaler, S. D. 1995, ApJ, 445, L141

Dreizler, S., Werner, K., \& Heber, U. 1995, in 9th European Workshop on White Dwarf Stars, ed. D. Koester \& K. Werner (Dordrecht: Kluwer), 160

Hill, J. A., Winget D. E., \& Nather, R. E. 1987, in IAU Colloq. 95, Second Conference on Faint Blue Stars, ed. A. G. D. Philip, D. S. Hayes \& J. Liebert (Schenectady: Davis), 627

Iben, I., \& Tutukov, A. V. 1984, ApJ, 282, 615

Kawaler, S. D. 1987, in IAU Colloq. 95, Second Conference on Faint Blue Stars, ed. A. G. D. Philip, D. S. Hayes \& J. Liebert (Schenectady: Davis), 297

\section{REFERENCES}

Kawaler, S. D., \& Bradley, P. A. 1994, ApJ, 427, 415

Kawaler, S. D., Hansen, C. J., \& Winget, D. E. 1985, ApJ, 295, 547

Kawaler, S. D., et al. 1995, ApJ, 450, 350

Kepler, S. O., et al. 1995, BaltA, 4, 221

Nather, R. E., Winget, D. E., Clemens, J. C., Hansen, C. J., \& Hine, B. P. 1990, ApJ, 361, 309

O’Brien, M. S., Clemens, J. C., Kawaler, S. D., \& Dehner, B. T. 1996, ApJ, 467, 397 (OCKD)

O'Brien, M. S., \& Kawaler, S. D. 1998, in preparation

Vauclair, G., Pfeiffer, B., Grauer, A. D., Belmonte, J. A., Jimenez, A., Chevreton, M., Dolez, N., \& Vidal, I. 1995, A\&A, 299, 707 (VPG)

Winget, D. E., Hansen, C. J., \& Van Horn, H. M. 1983, Nature, 303, 781

Winget, D. E., et al. 1991, ApJ, 378, 326

Winget, D. E., Robinson, E. L., Nather, R. E., Kepler, S. O., \& O’Donoghue, E. 1985, ApJ, 292, 606 